\title{
Epidemiological Perspective in Managing Viral Diseases in Animals
}

\author{
Mahendra Pal Yadav, Raj Kumar Singh, \\ and Yashpal Singh Malik
}

\begin{abstract}
Since the first report of a viral disease associated with plants, the fascinating field of virology has evolved and aided mankind altogether. Viral infections are known for inflicting colossal economic losses worldwide in food/work/companion animals. During the last few decades, emergence of a number of new viral diseases in animals, humans and plants has been visualized. Animal disease surveillance and monitoring is essential for the sustainability of healthy livestock production systems internationally. Preparedness for combating the emerging, re-emerging, exotic and transboundary diseases requires comprehensive monitoring and precision detection systems that are pliable under the field situations. With collective and concerted scientific interventions, a few of the animal viral diseases have been stamped out globally or regionally. Rinderpest, popularly called cattle plague, was eradicated from India in 2006 and globally in 2011. Notably, India achieved the disease-free status by OIE in 2014 for African horse sickness (peste equine), a deadly viral disease of equines. Likewise, equine infectious anaemia (EIA) and equine influenza (EI) have been controlled to a greater extent in India by adopting surveillance and monitoring along with zoo sanitary measures. Overall, there is a need for developing the 'One World, One Health' concept using multidisciplinary, regional and international networking to control major
\end{abstract}

\footnotetext{
M. P. Yadav

ICAR-Indian Veterinary Research Institute (ICAR-IVRI), Izatnagar,

Uttar Pradesh, India

Sardar Vallabhbhai Patel University of Agriculture and Technology, Meerut, India

R. K. Singh

ICAR-Indian Veterinary Research Institute (ICAR-IVRI), Izatnagar, Uttar Pradesh, India

Y. S. Malik $(\bowtie)$

ICAR-Indian Veterinary Research Institute (ICAR-IVRI), Izatnagar, Uttar Pradesh, India
} 
economically important emerging/re-emerging infectious diseases of humans and animals. This chapter describes various strategies for combating viral diseases of livestock.

\section{Keywords}

Virology · History · Livestock diseases · Diagnosis · Epidemiology · Disease eradication $\cdot$ Animal health programme $\cdot$ Control $\cdot$ Vaccines

\subsection{Prologue}

Ever since the identification of the causative agent of tobacco mosaic disease in plants, as a filterable agent (later named as 'virus') by D. Ivanovsky, a Russian scientist in 1892, marking the beginning of the science of 'virology', the fascinating field of virology has progressed and benefitted mankind globally. Subsequent to the isolation of the first plant virus, namely, tobacco mosaic virus (TMV), the first animal virus - foot-and-mouth disease (FMD) virus - was reported in 1897 by Loeffler and Frosch (1898). The virus aetiology of yellow fever in humans was established in 1900 by Walter Reed (Reed et al. 1901). In India, animal virology started around 1900 with research work on rinderpest (cattle plague) at the Imperial Bacteriological Laboratory, Mukteswar (now Indian Veterinary Research Institute), Izatnagar/ Mukteswar. One of the most important achievements for Indian animal virologists was the development of vaccines that proved efficient and safe. These were used for rinderpest and African horse sickness (AHS) eradication programmes (Yadav 2011; Yadav et al. 2016) and control of important viral, bacterial and parasitic diseases of livestock and poultry.

The creditable animal disease surveillance is critical for the sustainability of healthy livestock production systems of any country globally, as the threat of infectious diseases in the climate change scenario is large, diverse and dynamic which adversely affect the socio-economic conditions and welfare of livestock farmers/keepers (Malik et al. 2018). Preparedness for combating the emerging, re-emerging, exotic and transboundary diseases requires sound monitoring and precision detection systems that are cost-effective, flexible and adaptable under prevailing field conditions. There is also an international obligation for OIE (World Organization for Animal Health) reportable diseases of major significance in the trade of animals and animal products globally by all member countries of the World Trade Organization (WTO).

\subsection{Epidemiological Perspective}

Animal viral diseases inflict heavy economic losses globally in livestock, poultry and humans. The term 'epidemiology' originated in the last part of the nineteenth century from the Greek word 'epidēmia', meaning 'the knowledge of the prevalence of disease'. Different definitions have been coined for 'epidemiology'. The simplest 
definition describes epidemiology as 'the scientific study of the spread and control of disease in populations'. In other definitions, it has been described as the 'branch of medicine which deals with the incidence, distribution, and possible control of diseases and other factors relating to health' or 'the study and analysis of the distribution and determinants of health and disease conditions in defined populations'. Epidemiology has been classified as descriptive epidemiology, analytical epidemiology, spatial epidemiology, landscape epidemiology, temporal epidemiology, local and global epidemiology, molecular epidemiology and applied epidemiology. The data collected on the epidemiology of important livestock diseases is of immense use in formulating appropriate disease control and management strategies.

The technological advances made in proteogenomics and immunomics have showed the way for understanding the genetic basis of host-pathogen interactions influencing the host immune response. New proteomic approaches including T-cell and B-cell epitope mapping have given a boost to the pace to discover antigen-antibody relationships, thus giving a push to the development of newer diagnostics and vaccines for infectious diseases. Innovations in genomic technologies have paved the way for unravelling interactions between the microorganisms and cells of the innate immune system. The advent of molecular techniques in recent decades has made visible impact on the study of the epidemiology and resultant boost to the understanding of disease dynamics, aetiology, diagnosis and charting of suitable control measures at faster speed. A large number of molecular techniques have been developed and used to address epidemiological concerns. Different techniques are now available for different aspects of investigations (Joshi et al. 2013). At the core of 'molecular epidemiology' is the need for high accuracy and specificity in typing the disease-causing agents; to monitor the spread of pathogens in populations and different species and regions; to trace back the original source of the causal agent; to study variations in the antigenicity, pathogenicity and immunogenicity; to differentiate between enzootic and panzootic infections; to discern the mode of transmission of the causative viral agent from host to host; and to sense the existence of strain variants in the vulnerable population and/or individual, besides addressing other epidemiological parameters and issues (Chakraborty et al. 2014; Singh et al. 2017). Availability of newer innovative molecular technologies in recent years has revolutionized the study of patho-immunobiology and understanding the disease dynamics, leading to better diagnostics and vaccines. Molecular subtyping, being more discriminating, is considered to be better than most of the phenotypic subtyping methods as it is least influenced by the organism's responses to environmental factors.

The interactions between the host, pathogen and environment are known to influence the epidemiology of the disease. Numerous factors are known to influence the epidemiology of disease, such as sex; age; nutrition; immune status of the host; climatic conditions (heat, cold, humidity, wind velocity, ambient temperatures); role of vectors; survival of virus in nature; duration and extent of excretion of virus from infected host; reservoirs/carriers of the infectious agent; susceptibility of the pathogen to common disinfectants; reactivation of latent viral infection under immune suppression; vaccination campaigns; presence of naturally occurring attenuated strain of virus in the population; spillover of the infectious agent/virus from their 
natural niches due to encroachments; developmental projects for laying railway tracts, roads, etc., leading to mixing of human and domestic animal populations with wildlife; ecological changes on account of deforestation and making dams, canals, etc.; antigenic multiplicity/stability of the virus; intimate contact of humans and livestock with wildlife/wildlife products; social/religious customs; and extent of the availability of funding for capacity building.

\subsection{Diagnosis of Viral Diseases of Livestock}

Viral infections have been identified as an important cause for inflicting huge losses worldwide in food/work/companion animals, including sheep, goats, cattle, buffaloes, equines, camel, yaks, mithun, canines, pigs, poultry and fish. In the last 40 years, the world has witnessed the emergence of a number of new viral diseases in animals, humans and plants in various parts of the world with more severe consequences due to ecological, demographic and climatic changes. While about $60 \%$ of the infections are zoonotic, $75 \%$ of new viral diseases reported during the last three decades are zoonoses, i.e. transmissible between animals and humans. Interplay of host, pathogen and environment-related factors in the epidemiology of diseases justifies to cover viral and other infections under the 'One World, One Health' umbrella. Timely detection and accurate diagnosis of the viral aetiologies allows better selection and adoption of appropriate and timely management practices including prophylactic vaccination of the susceptible population or therapeutic vaccination in the affected livestock population. Therefore, development/standardization of diagnostic techniques which are reliable, time-efficient, cost-effective, sensitive, specific and feasible under field conditions is of utmost importance for prevention, control, eradication, monitoring and forecasting of infectious and contagious diseases (Dhama et al. 2014). Diagnosis of viral infections has greatly advanced in recent decades with the use of state-of-the-art technologies, using modern biotechnology, nanotechnology and molecular biology.

For the diagnosis of animal viral diseases, both conventional and molecular tools as well as new-generation diagnostic techniques are employed. Apart from observing clinical signs, postmortem lesions and histopathology, isolation and identification of the viral agent employing in vitro cell culture techniques and embryonated chicken eggs, in vivo isolation in the host animals and demonstration of the virus particles by electron microscopy or viral proteins/nucleic acid in tissue sections or infected cells using immunofluorescence/immunoperoxidase technique are also utilized. Other conventional tests include haemagglutination, haemagglutination inhibition (HI), haemadsorption, haemadsorption inhibition (HADI), agar gel immunodiffusion, counterimmunoelectrophoresis, enzyme immunoassays, latex agglutination test (LAT), etc. These conventional disease diagnostic techniques are time consuming and laborious, and some of these even require in vivo systems. Moreover, it is difficult to differentiate antigenic variants and virulent strains from classical strains by conventional methods. Therefore, it has been emphasized time and again to develop newer diagnostics with improved sensitivity and specificity which can also 
differentiate newly evolved pathogen types from classical or vaccine strains (Dhama et al. 2014).

Advances in molecular biology and recent knowledge of virus pathogenesis have paved the way for the development of highly sensitive and specific nucleic acidbased detection systems for many viral diseases. By using advanced state-of-the-art modern tools, the detection of animal pathogens has become more reliable and rapid. Molecular tools and techniques are commonly used nowadays for detection, differentiation, characterization, monitoring, pathogenicity study, and analysis of epidemiological status to assess the genomic relationship or variations and tracing the probable origin of viral pathogens. The nucleic acid- and antigen detectionbased molecular techniques are gaining preference over the conventional diagnostic tests based on demonstration of specific antibody in the serum or antigen(s) in the tissues of the host species. The polymerase chain reaction (PCR) and its variations (RT-PCR, real-time RT-PCR with TaqMan, multiplex real-time PCR, nested RT-PCR, q-PCR, RAPD-PCR, REP-PCR), RFLP, RISA, SNP, SNR, VNTR, AFLP, polymerase spiral reaction (PSR), PCR-ELISA (enzyme-linked immunosorbent assay), loop-mediated isothermal amplification (LAMP), surface plasmon resonance (SPR), sensor-based microarray DNA chips, immuno-biosensors, nextgeneration sequencing (NGS), mass spectrometry, genomic hybridization, nucleic acid probes, sequence-based typing, single nucleotide polymorphism, nucleotide sequencing, phylogenetic analysis and whole genome sequencing are used with precision. Techniques like real-time reverse transcription PCR (RRT-PCR) have made it possible for real-time detection and allowing confirmation of virus within a couple of minutes.

Further, advances in biomedical instrumentation techniques and nanobiotechnology have led to the development of microarray, biochips and biosensor platforms that have revolutionized the modern-day diagnostics, and fully automated small micro devices have become a reality for providing instant 'point-of-care' (PoC) diagnosis (Rout et al. 2018). Apart from being very sensitive, specific and quick, these can also be used even if the pathogen has lost infectivity. With these techniques it is possible to differentiate closely related organisms directly from clinical samples. These technologies can be used to pinpoint the origin/evolution of the pathogen, making them very powerful tools for studying epidemiology. The present era also demands highly sensitive, specific, rapid, cost-effective, labour-friendly and off-the-shelf, pen-side diagnostic assays for diagnosing metabolic disorders and infectious diseases. With a plethora of decisive advantages, the nanodiagnostics are proving to be a promising substitute to in-use diagnostic techniques. The prospective applications of nanodiagnostics are manifold. To name a few, these have an edge in the area of detecting infectious agents, tumours, intracellular and tissue imaging, immunohistochemistry, multiplexed diagnostics and fluoroimmunoassays. The increasing use of quantum dots, decorated gold nanospheres and nanoshells, nanobarcodes and nanobiosensors in the field of diagnostics is quickly taking over diagnostic techniques of the past as these provide accurate, faster and sensitive monitoring and surveillance tools and intensify network approaches assisting greatly in formulating effective disease prevention and control strategies. The 
microfluidic technology offers as a cost-effective substitute for disease diagnosis in the field.

The nanodiagnostics have a promising future to shift the paradigm from organized laboratories and skilled personnel to point-of-care testing and lab-on-chip technologies, which are user-friendly and can provide instant diagnosis right at the doorstep of livestock owners. One of the prerequisites for developing any farmerfriendly diagnostic assay is to use the reagents that do not require any cold chain facilities. In this direction, colloidal nanogold particles fabricated with either antigens or antibodies have been used for developing field diagnostic assays for animal diseases. The immuno-comb assay has been developed for rapidly detecting PPR virus-specific antibodies in serum samples. Similarly, colloidal gold particles fabricated with antibodies have been used to detect the PPR virus in a lateral flow assay. Both these diagnostics can be used at the doorstep of farmers without requiring the use of any sophisticated instrumentation. Recently, peptide, nucleic acid and colloidal gold nanoparticle-based visual diagnostic assay has been developed for Newcastle disease virus.

To keep pace with the recent advancements in the diagnostic arena, researchers are working on biosensor-based diagnostic platforms. In this direction, research work on the development of surface plasmon resonance (SPR) optical sensor-based label-free diagnostic assays has been initiated, and biosensor assays for detecting PPR virus and specific antibodies in clinical samples have been optimized recently (Rout et al. 2018). The technique is advantageous because it can quantitatively detect the target in real time within less than 10 min and also can automatically analyse a number of samples in high-throughput manner to provide rapid and confirmatory diagnosis of this disease. Efforts need to be directed towards developing this type of label-free biosensor assays for other viral diseases of livestock also.

Among the antigen/antibody detection-based tests, ELISA and its modifications, namely, DIVA C-ELISA, IC-ELISA, sandwich ELISA, strip ELISA, dot ELISA, immune stick ELISA, liquid-phase blocking ELISA and pen-side diagnostic kits, have advanced the identification and management of viral infections globally. The metagenomic approach has opened a unique method for the detection of hitherto unknown/unexpected infectious viruses, variants of existing viruses and other pathogens. Recently, several new novel viruses, namely, bocaviruses, torque teno viruses, astroviruses, rotaviruses and kobuviruses, have been identified in porcine disease syndromes. Nowadays, in veterinary diagnostic virology, the metagenomic approach of detecting viral pathogens is becoming a useful cultivation-independent tool.

For accurate and rapid diagnosis, capacity building of diagnostic laboratories adopting GLP, trained human resource, biosafety and containment facility according to the category of the virus being handled in the laboratory are prerequisite for checking spillover of the infectious agents from the laboratory to the environment or posing risk to the laboratory staff, when dealing with zoonotic agents. Development of indigenous diagnostic reagents, kits, vaccines and DIVA testcompliant vaccines is required for cost-effectiveness and better immune response and interpretation of vaccinal immunity and efficacy of mass vaccination campaigns in hand (Rout et al. 2014). Harmonization of the diagnostic tests, reagents and SOPs 
between the laboratories in networking mode is a must exercise to be followed for comparative results. Development of thermo-resistant vaccines will have added advantage in tropical countries having problems in maintaining cold chain in rural areas. Thus, as far as possible, innocuous reagents and reverse genetics should be used for the development of diagnostic tests and/or vaccines.

\subsection{Animal Disease Monitoring and Surveillance Database}

In India, the ICAR-National Institute of Veterinary Epidemiology and Disease Informatics (NIVEDI), Bengaluru, is responsible for livestock disease informatics, seromonitoring of important livestock diseases, forecasting and forewarning, as well as to assess the economic losses due to animal diseases. An innovative epidemiological software, the National Animal Disease Referral Expert System (NADRES), was developed at the institute as a web-based dynamic and interactive disease relational database supported by geographic information system (www.nadres.res.in.). To suit the needs of veterinary epidemiology in India, EpiInfo@ software of CDC Atlanta, USA, has been considered. An access-based software for 'National Livestock Serum Repository' (India.admasEpitrak - an exclusive epidemiology offline software) has also been created at this institute.

The epidemiology of important livestock diseases is studied by the institute and used in formulating appropriate disease control strategies. Furthermore, they have also established a few spreadsheet modules to assess the economic impact of certain abortive diseases. Some of the other significant achievements include development of a forecasting module for predicting possible occurrence of nationally important viral and bacterial diseases of livestock 60 days preceding the likely outbreak (Anonymous 2011, 2013), identification of eco-pathozones for the economically important livestock diseases, development of bluetongue disease map in the endemic states of Karnataka and Tamil Nadu, and systematic seroprevalence studies on infectious bovine rhinotracheitis (IBR) and peste des petits ruminants (PPR) in animals.

The livestock sector in India, known for its magnificent animal wealth, has been recognized as a potential solution for addressing the national nutritional insecurity and an ideal platform for addressing the unemployment problem in women and youth. Livestock rearing is central to the livelihoods and nutritional security of millions of small and marginal farmers and landless agricultural labours across the country. India is blessed with rich livestock resources with diverse species, breeds and strains and impressive production performance. It has the world's largest bovine dairy herd of around 300 million comprising of cows and buffaloes and stands first in milk production globally since about two decades (1998 onwards). Among the many areas of concern that limit the realization of the full potential of the livestock sector, the rising and unescapable outbreaks of viral diseases among animals are posing considerable challenges to livestock health and production. Although exact estimates due to various viral diseases to livestock industry are not available due to inadequate reporting of the disease outbreaks, the viral diseases are most important as they cause heavy economic losses through morbidity, mortality and other direct 
and indirect costs on treatment, hygiene, disinfection and sanitary measures; loss in production, reproduction and working capacity of animal; and replacement costs of stock. Unlike bacterial, fungal, parasitic and mycoplasma diseases, non-availability of cost-effective antiviral drug therapy, rapid spread, etc., make the task of their control more difficult.

Over the past few decades, many of the bacterial and viral diseases have negatively impacted the socio-economically deprived people, sustaining mainly on livestock. Agricultural activities including ploughing and tilling of the fields and agricultural product transportation suffer adversely due to the assault of viral diseases. With the collective and concerted scientific interventions and enabling policy support, a few of the animal viral diseases have been stamped out globally or regionally. Rinderpest, popularly called cattle plague, an ancient viral disease of bovines, caprine and swine was eradicated from India in 2006 and globally in 2011 (Yadav 2011). India has been given disease-free status by OIE in 2014 for African horse sickness (peste equine), a deadly viral disease of equines. Equine infectious anaemia (EIA) and equine influenza (EI) have been controlled to a greater extent in India by adopting surveillance and monitoring along with zoo sanitary measures (Singh et al. 2018).

\subsection{Success Story of Eradication/Control of Animal Viral Diseases}

\subsubsection{Rinderpest}

Rinderpest was once a serious threat to the livestock industry in several regions of the world, especially in Asia, Africa, Europe and the Americas. The infection and death rates in newly exposed naïve population were as high as 95-100\% leading to colossal economic losses. A death rate of around 200,000 animals per annum was recorded among the affected bovine population of 400,000 during the first half of the 1950 s in India. Throughout the history of mankind, the social, economic and ecological consequences due to rinderpest had been more serious and severe. In India, the presence of rinderpest was confirmed by the Cattle Plague Commission (Hallen et al. 1871). This disease has been conquered successfully by following mass vaccination along with zoo sanitary measures. The FAO declared the global eradication of rinderpest on 28 June 2011, marking it as the first ever viral disease of animals eradicated globally about three decades after the eradication of small pox, a viral disease of humans in 1980 (Yadav et al. 2016). In India, dividing the country into four zones based on the epidemiological picture of the disease and adopting strategic and focused vaccinations at interstate and international borders and migration routes of bovines and caprine for creating immune belts, coupled with rigorous clinical and serosurveillance, were of great help in achieving freedom from the infection. With the successful eradication of rinderpest, the livestock sector across the globe became safer and consequently the livings of livestock farmers improved.

Eradication of rinderpest helped in assuring inclusive growth as it mainly benefitted landless, marginal and smallholder livestock keepers, besides providing much 
needed animal protein, food and nutrition security and livelihood security. The milk production in India enhanced 2.99 times from the year 1955 to 1995 and further stepped to 4.796 times by 2006. Similarly, the bovine meat production increased by 17.99 times between 1959 and 1995. The income from bovine milk and meat increased to 102.06 and 193.96 times, respectively, between 1950-1951 and 20052006. In value terms these benefits amounted to 15563.56 million US dollars in respect of milk and 435,011 million US dollars for bovine meat from the year 1950 to 1996. It is estimated that India gained additional food production valuing 289 billion US dollars from 1965 to 1998 due to reduction in rinderpest incidence. This is one of the greatest contributions of veterinary scientists to crop production and dairy development programmes in India after Independence (Uppal 2011).

The success of rinderpest control and eradication proved a rewarding experience and landmark for the veterinary services in India, providing confidence and capacity building to undertake a control programme of livestock diseases at the national level. The freedom of the country from rinderpest not only enabled the growth of the dairy industry in India but has also boosted the export of meat and other dairy products in recent decade. Today India tops not only in milk production in the world but also the largest exporter of buffalo meat. Cost-benefit analyses indicated that every dollar spent on rinderpest control programme gained about 20 dollars to the Indian dairy industry through more milk, meat and draft power for better agricultural productivity (Uppal 2011).

\subsubsection{African Horse Sickness}

African horse sickness (AHS) is a devastating, highly infectious, non-contagious, insect (biting midge)-transmitted viral disease of equines. After the detection of the first animal virus (foot-and-mouth disease virus), AHS virus was the second animal virus discovered by John McFadyean at the Royal Vet College London in blood samples from Africa. This virus affects all species of Equidae family including horses, mules, donkeys and zebras. In susceptible horse population, the consequence of AHS can be dreadful, resulting in up to $95 \%$ mortality. At present as there is no treatment available against AHS virus, vaccination is the only weapon available against this dreaded disease. On 27 May 2014, India touched a major landmark by getting official disease-free status from African horse sickness (peste equine). As per Resolution No. 19 (82nd General Session), India was declared as member country recognized free from African horse sickness according to the provisions of Chap. 12.1 of the Terrestrial Code by the World Organization for Animal Health (OIE) (82 GS/FR - PARIS, May 2014).

\subsubsection{Animal Viral Disease Control in India}

Trade of livestock and livestock products, within and between countries, has resulted in enhanced risk of spread of diseases to livestock, poultry and human beings. The 
occurrence of highly contagious viral diseases of livestock and poultry, namely, foot-and-mouth disease (FMD), peste des petits ruminants (PPR), classical swine fever (CSF), bluetongue, equine infectious anaemia (EIA), equine influenza (EI), highly pathogenic avian influenza (HPAI) A virus, infectious bursal disease (IBD) and zoonotic diseases such as West Nile fever, Rift Valley fever, SARS-coronavirus, Nipah virus infection, Hendra virus, swine influenza virus A (H1N1) and CrimeanCongo haemorrhagic fever (CCHF), has compelled to formulate policies and regulatory procedures to prevent the entry of transboundary and exotic diseases on the one hand and interstate spread of livestock diseases through uncontrolled movements of animals for work, migration, grazing, etc., on the other hand, for checking the dissemination of endemic diseases within the country.

For the planning and execution of control programmes against viral disease, it is necessary to fully understand the disease, particularly the interaction between the host and the pathogen as well as between the pathogen and the host vis-à-vis the environment. This knowledge should include the duration of incubation period; pathogenesis; route of entry of virus in the host species; extent and duration of excretion of the virus from the host; reservoir and carrier hosts; duration and mechanism of interepidemic survival of the virus; survival of the virus in nature including its susceptibility to high and low temperature, freezing and thawing, acid and alkaline $\mathrm{pH}$ and disinfectants; survival in body fluids; and antigenic variations, such as types, subtypes, clades and genotypes, particularly in RNA viruses. Appropriate knowledge of these parameters will be very useful in planning and implementing cost-effective disease control programmes. For example, in case of equine influenza, the knowledge that the virus is excreted for not more than 10 days from the infected animal can be used with advantage to check the spread of infection by restricting animal movements from infected to healthy premises and vice versa for 2 weeks. Similarly, all direct and indirect contacts between the sick and healthy animals and farms need to be avoided, besides symptomatic palliative treatment and complete rest to the sick animal to avoid secondary bacterial complications. In case the fever lasts for more than 3 days, antibiotic therapy should be considered to combat secondary bacterial infections. A three-day rest is recommended for each day of fever the animal had run in equine influenza. Influenza virus is highly susceptible to freezing and thawing and common disinfectants, such as Dettol, Savlon, 70\% alcohol, phenyl, $\mathrm{KMNO}_{4}$, ultraviolet light and sunlight, but fairly resistant to sodium carbonate $\left(\mathrm{Na}_{2} \mathrm{CO}_{3}\right)$ and sodium hydroxide (Yadav et al. 1993). Thus, suitable cost-effective disinfectants may be used for decontamination of the stables and adjoining premises, ropes of animals, hands, shoes and clothing of animal attendants. The outbreaks of influenza, which are more common during winters, fade away in summer due to the susceptibility of the virus to high ambient temperatures.

Equine infectious anaemia (EIA), caused by a retrovirus of Lenti group, is transmitted by blood-sucking insects, including mosquitoes and flies. The virus remains lifelong in latent form in animals which survive from the disease. The disease was reported in India for the first time in 1987 (Uppal and Yadav 1989). It can relapse due to immunosuppression on account of stress conditions due to pregnancy, hard 
work, cortisone therapy or other factors. Such carrier animals are thus potential source of infection for spreading the disease throughout their life. In the absence of a suitable vaccine, the only solution to limit the infection is by destruction of seropositive animals. However, in the absence of proper legislation, the owners did not agree to put down their EIA-positive animals immediately and have to be convinced about the accuracy and validity of the test by repeat tests of the animals on fresh serum samples in the same laboratory or at other laboratories in the same country or abroad. The authors, while confronted with this problem in India, made use of an old observation of a researcher wherein it was shown that an insect after feeding on an EIA-affected equine does not go beyond 200 meters and prefers to come back to the same host for the next blood meal, by isolating the seropositive animal in Coggins test (agar gel precipitation test) more than 200 meters away from other equines before they were put down. This method was employed on hundreds of EIA-seropositive animals and always found effective.

\subsubsection{Foot-and-Mouth Disease Control Programme}

After successful eradication of rinderpest, foot-and-mouth disease (FMD) of cloven-footed animals is another OIE-listed important viral disease inflicting heavy economic losses and adversely affecting the trade of livestock and livestock products from India to other countries. Direct losses due to FMD in India have been estimated to the extent of INR 2,30,000 million per annum. Accordingly, to combat FMD, the Government of India (GoI) initiated the FMD Control Program (FMD-CP) in 2003-2004 during the 10th Five Year Development Plan in 56 select districts of seven states having bearing on milk production. The FMD-CP envisaged vaccination of cattle and buffalo population using indigenously produced killed adjuvanted trivalent $(\mathrm{O}, \mathrm{A}$ and $\mathrm{Asia} 1)$ vaccine under nationally coordinated and monitored mass vaccination programme following OIE progressive pathway. Encouraged with its success in reducing the incidence of the disease, the programme was expanded to further 221 districts in 14 states in the 12th Plan. Now, since 2016 the entire country has been covered under FMD-CP with the ultimate objective to eradicate the disease by 2040. FAO/OIE have targeted to control FMD by 2035 . This will be followed by stopping vaccination and conducting surveillance for freedom from clinical disease followed by freedom from infection. These activities are expected to be over by 2040. The control programme involving six monthly rounds of vaccinations has shown encouraging results as reflected in reduction in the number of outbreaks and incidence of the disease. The number of outbreaks declined from 1911 in 2003 2004 to 149 in 2017-2018 reflecting about 92\% drop in FMD incidences. The years 2016-2017 and 2017-2018 were mainly dominated by serotype 'O' FMD virus as there were 296, 03 and nil outbreaks due to serotypes 'O', 'Asia1' and 'A', respectively (Anonymous 2017-18).

\subsubsection{PPR Control Programme}

Subsequent to the detection of PPR in the southern peninsula in India in the late 1980s, the disease became widespread and endemic by 1995-1996. The disease is estimated to cause global losses between 1.45 billion and 2.1 billion US dollars per 
year. The disease causes economic losses in India to the tune of INR 11070 million per year (Tripathi et al. 2018). After developing diagnostic facilities and a safe and potent vaccine indigenously by the year 2001-2002, a national control programme on PPR (NCP-PPR) was started in 2010 in five states (Kerala, Tamil Nadu, Karnataka, Andhra Pradesh, Maharashtra) and five union territories (UTs). During February 2014, the programme was extended to all the states and UTs. The aim of the programme is to undertake intense immunization of sheep and goats and their three subsequent generations. With judicial use of vaccine and diagnostics, the NCP-PPR has shown encouraging results in Karnataka, Andhra Pradesh and Chhattisgarh with 75\% reduction in the disease incidence (Tripathi et al. 2018). India is expected to attain infection-free status of the disease within the time frame of 2030 set by FAO and OIE for eradication of PPR in small ruminants from the globe.

\subsection{Zoonotic Viral Diseases}

Viral zoonotic diseases of animal origin also pose threat to human welfare and livelihood through morbidity, mortality, reduced nutrition and working capacity. The explosive growth in human population, increasing urbanization, high density of livestock and poultry populations in modern livestock farms, environmental degradation, deforestation, contact with wildlife and climatic changes are some of the factors responsible for emergence of zoonotic diseases in recent decades (Dhama et al. 2018). The chances of a spillover of a pathogen from domestic or wildlife species are more in countries where the public health infrastructure is suboptimal and the interaction between humans and animals is more intimate. The hotspots for infectious disease emergence are generally places where wildlife, livestock and human interactions are more frequent. This is best exemplified by the emergence of Nipah virus in Malaysia and SARS in Guangdong Province of China. As per a report (Mapping of Poverty and Likely Zoonoses Hotspots, 2012) from the International Livestock Research Institute (ILRI), Ethiopia, Nigeria, and Tanzania (Africa) and India (Asia) exhibit the highest disease burden of zoonoses, with parallel illness and death. The zoonotic diseases cost US \$ 6.7 billion a year worldwide.

There are more than 1000 known animal pathogens, of which about $40 \%$ pathogens of domesticated livestock species and 70\% of domestic carnivores have zoonotic potential. Though at present only $11-18 \%$ of zoonotic pathogens from domestic livestock and carnivores are viruses, a significantly high number (55-59\%) of emerging zoonotic pathogens are viruses. Approximately, 90 novel human pathogens were discovered during the last 30 years, averaging 3 per year, $66 \%$ of which were viruses, and more than $80 \%$ of these are RNA viruses. The propensity for emergence of new variants of RNA viruses is very high because of their small and segmented genome, rapid rate of multiplication and polymerase enzymes that lack proofreading capability. As a result, these viruses are more prone to exchange 
genetic material from related viruses when coinfecting the host by recombination or reassortment events. Pathogens that can infect multiple species and those that find a closely related host species in close proximity can jump species under suitable conditions and may cause the emergence of a new disease which may lead to epidemics/pandemics. Bovine spongiform encephalopathy (BSE) and scrapie, caused by prions, are examples of species jumping. Feeding of cows on scrapie-infected sheep offal and meat meals in the UK resulted in the development of BSE or mad cow disease. Subsequently, consumption of beef from BSE-affected cows in people developed another version of this disease, namely, Creutzfeldt-Jakob disease.

\subsubsection{Combating Zoonotic and Non-zoonotic Viral Diseases}

In the present scenario of constantly increasing worldwide population, urbanization; globalization; industrialization; mutable lifestyles and food habits; tourism; intensified animal farming; ecological and biodiversity changes; emerging antimicrobial/drug resistance, coupled with lack of effective, safer and cost-effective treatment regimens and vaccines; immunosuppression due to multiple factors; and global warming are resulting in higher susceptibility of the host to the pathogen(s) and vaccine failures. This emphasizes the development of the 'One World, One Health' concept using multidisciplinary, regional and international networking to counter economically important, emerging/re-emerging infectious diseases of humans, livestock and poultry. The important infectious diseases/pathogens to be tackled include FMD, PPR, coronavirus, rotavirus, parvovirus, infectious bovine rhinotracheitis (IBR), bluetongue, toroviruses, infectious bursal disease (IBD), avian influenza, Newcastle disease (ND), Marek's disease (MD), avian infectious bronchitis (IB), chicken infectious anaemia (CIA) and hydropericardium syndrome. Additionally, there is a need to counter several pathogens of zoonotic significance including rabies virus, Rift Valley fever, West Nile virus, avian influenza A (bird flu), swine influenza A virus (H1N1), viral encephalitis, Crimean-Congo haemorrhagic fever (CCHF), Hendra virus, Nipah virus, Ebola and Zika viruses.

There is also a need to monitor wildlife, migratory birds and important vectors and reservoirs of various infectious agents having a role in the maintenance/survival and spread of the pathogens. Recent threats of epidemic/pandemic nature of severe acute respiratory syndrome (SARS), highly pathogenic avian influenza (HPAI) A virus (H5N1) and swine influenza A virus (H1N1) warrant strengthening of global health issues through capacity building for enabling comprehensive preventive and control measures. Besides this, preparedness and prompt response are a must for successful control and management of the devastating diseases posing grave threats to humans as well as to food and companion animals and posing immense socioeconomic burdens. For the efficient management and control of viral zoonoses, well-planned strategic approaches and interventions through collaborative working of medical and veterinary professionals are needed. 


\subsection{Networking of Animal Health Programmes}

The advent of geographical information system (GIS) has proved of great help for efficient disease detection, reporting and recording among populations, cluster analysis, spread of infections and their modelling, assessment of outbreak magnitude and planning of efficient and effective control strategies. The GIS is of immense help for mapping the location of herds/flocks and other related parameters. Use of GIS has greatly facilitated the knowledge of epidemiologists, diagnosticians, clinicians and researchers. Multidisciplinary efforts are required to realize the 'One World, One Health' concept at global, national and local levels in the overall interest of our society. A number of crucial issues at the global level, such as rapid population growth, emerging antibiotic resistance, climate change and global warming, international trade and travel, food safety, migration of people from rural to urban areas, biosecurity and biosafety concerns, ecotourism and disease surveillance and monitoring, have underlined the importance of networking of these programmes for faster solutions. Increasing risk for the emergence/re-emergence of deadly/debilitating zoonotic diseases including rabies, highly pathogenic avian influenza virus, swine influenza A (H1N1) virus, Nipah virus infection, tuberculosis and brucellosis has led to advancement of novel diagnostic techniques and vaccines and contributed immensely to human and animal health impacting global health as a whole. FAO of the United Nations has established linkages with various funding institutions at regional levels in various parts of world through GIS. It ultimately led to engagement of manpower as well as investment for strengthening of veterinary services along with the whole spectrum of disciplines leading to the protection and promotion of animal health.

Efficiency of control programme for viral diseases will depend on several factors, such as rapid and accurate diagnosis of the disease, incidence and/or prevalence, molecular epidemiology of the disease to understand antigenic variation at protein or gene level, choice of the diagnostic test and stage of the disease, correct interpretation of the test results and decision regarding undertaking control and preventive measures including vaccination, disinfection, stamping out, type of vaccine (live modified, killed, adjuvanted), adjuvant used in the vaccine and duration of immunity, route of vaccination, extent of humoral and CMI response induced, frequency of booster vaccination, affordability and availability of vaccine and cold chain availability under field conditions. For ease of vaccination and reducing the stress to the animals and the vaccinator in controlling the animals, in future combined vaccines, multiple vaccines - peptide, split, thermostable, and recombinant vaccines - will be more in demand. Similarly, intradermal and pressure vaccination may become a common practice. Vaccination in advance stage of pregnancy should not be practiced to avoid immune tolerance in the newborn. Merits/demerits of in ovo vaccination in poultry also deserve due consideration, as the newborn chicks get antibodies from the mother hen through the yolk. 


\subsection{Journey Through Animal Viral Vaccine Development}

Infectious diseases are one of the most hazardous enemies mankind has faced as these are capable of destroying all his economic strengths by affecting their pets, domesticated animals and themselves. For profitable animal husbandry practices, prevention and control of diseases should be considered on cost-benefit ratio basis. The colossal losses are incurred during disease outbreaks like FMD, IBR, PPR, bluetongue, sheep pox, goat pox in livestock and IBD, MD, IB, EDS, CIA and avian influenza in poultry. Vaccines and judicial vaccination procedures are only ways to prevent and control such diseases in a cost-effective manner. The concept of vaccination was first popularized by Edward Jenner in 1798 as a method to fight against the deadly human small pox disease. However, prior to it, Chinese people were practicing a form of vaccination called 'ovination' where virulent sheep pox virus was inoculated in sheep to generate immunity against further infection with sheep pox virus. Similarly, in FMD, in the absence of a vaccine, 'apthization' (deliberately rubbing the infected fluid or lesions of diseased animals into the healthy animals of the farm/herd) was practiced with the objective of reducing the time, labour and cost towards the management of the outbreak in one go at the farm. The ILT virus, a herpes virus which causes respiratory disease in poultry, was recommended to be inoculated in the cloacal region to impart immunity in want of vaccine availability. However, now with the availability of dependable vaccines against most of the infectious diseases it is not advisable to use such methods as it has the risk of spreading the virulent virus to susceptible animals. After the successful initial approaches by Jenner using cow pox virus as vaccine against small pox in human, the concept of killed vaccine was made into use by Louis Pasteur in 1885 to prevent post-bite rabies disease in man. The mid-1940s designated as 'the era of cell culture' made a revolution in vaccine research to develop more attenuated live organisms as vaccine. Vaccine research has moved from the application of whole organism approach (inactivated or live attenuated) to synthetic antigenic peptide-based or gene-based vaccine approaches for man and animals against a number of infectious diseases.

Vaccine discovery, though initially made by chance due to keen observation or hit and trial method, later became a deliberate attempt to combat infectious diseases. In initial times the whole organisms were applied in scarified wounds to give the host an opportunity to mount an immune response against an infectious agent. But later more specific and safer killed or attenuated live organism-based vaccines became important in vaccine research even though the vehicles added to make the vaccines more immunogenic were causing serious health problems in the individuals given the vaccines. Availability of desired vaccines against the lethal, wasting and debilitating diseases has backed immensely the attainment of successful public health programmes. As of now, scientific challenges still exist to develop safer, effective and reliable vaccines that boost protection against the pathogens of major significance. With the recent advancement in the field of immunology and vaccinology, modern biotechnological innovative approaches are becoming available to counter such diseases. 
To ameliorate these problems, recombinant DNA technology-based vaccine research gained strength and advanced the idea of gene delivery as vaccines using shotgun method, replication-defective live virus vector vaccine, single-copy virus vaccine and split, peptide and edible vaccines have been researched. Thus, vaccinology has traversed through three successive phases/eras. The first one was with initial stages using scabs or infective material of related diseases in animals to prevent reinfection. The second era started with the advent of cell culture or chicken embryobased techniques and introduction of inactivated and attenuated viruses as vaccine, whereas the inclusion of rDNA technology in vaccine production made them to advance to the third era. The innovative technologies have transformed the concept of vaccine development and will go a long way in the generation of safer and more effective vaccines. Scientific advancements and their application will certainly provide better-quality health-based products. Based on cost-effectiveness, vaccination still remains a high priority for the prevention and control of animal diseases. These advancements in vaccine development will meaningfully lessen the prevalence of diseases in man and animals. An ideal vaccine is expected to have minimum threshold of the antigenic (immunogen) mass devoid of extraneous antigens and capable of inducing solid and long-lasting immunity.

\subsection{Handling of New Viral Disease Outbreaks}

When an exotic viral disease struck a country for the first time, it may initially affect one animal, few animals or a large number of animals. The strategy to be adopted for containing the outbreak will depend on the nature of the virus, its spread, risk assessment and country legislation on disease control and prevention. Thus, there is a need to develop strategic plans for the prevention and control of exotic and transboundary animal diseases on case-to-case basis. Examples of such viral diseases from Indian perspective include African swine fever; transmissible gastroenteritis (TGE) in pigs; swine vesicular disease; Rift Valley fever; West Nile fever; Eastern equine encephalomyelitis (EEE); Western equine encephalomyelitis (WEE); Venezuelan equine encephalomyelitis (VEE); AHS; FMD virus types 'C', 'SAT I', 'SAT II' and 'SAT III'; Nipah virus; Hendra virus; SARS-coronavirus; and prion diseases - bovine spongiform encephalopathy (BSE) and scrapie.

\subsection{Biosecurity Measures to Combat Viral Infections}

Institution of appropriate timely biosecurity methods is important for preservation and improvement of animal health in order to minimize the risks from infectious diseases. Lapses in biosecurity in the management of livestock and poultry are often responsible for higher incidence of infectious and zoonotic diseases of animals. This is more so in case of viral diseases of animals and poultry. Close contacts between animals, wildlife and humans will facilitate the spread of viral and other infectious diseases. The emergence of new viral diseases/infections, such as Rift 
Valley fever, West Nile fever, SARS-coronavirus, Hendra virus, avian influenza A H5N1, Nipah virus, Zika virus and swine influenza A (H1N1) virus, from time to time is a glaring example of zoonotic disease threats adversely affecting animal health and public health, national economies and food and nutrition security globally. Due to breach in the biosecurity, deadly viral diseases like FMD had re-surfaced in countries which were having disease-free status for decades. Disease incursions through imported livestock and poultry, fish and their products in the past in India have been responsible for the introduction of a number of diseases like PPR, bluetongue, IBR, IBD, CIA and classical swine fever (CSF) in India. AHS was responsible in the death of around 3,00,000 equines between 1959 and 1961 in Asia including India and other countries (Kumar 1976). Subsequent to its first occurrence in 1996 in China, the highly pathogenic avian influenza (HPAI) A virus H5N1 has affected more than 60 countries in Asia, Europe, Africa and North America. The virus affected wild birds as well as domestic poultry. Sporadic cases of transmission to humans in close contact of infected birds with sizeable mortality raised pandemic concern of 'bird flu'. Since the first reporting of H5N1 virus from India and Bangladesh in 2006 and 2007, respectively, both these countries are experiencing outbreaks almost every year. The zoonotic disease outbreaks due to animal pathogens underline their double-sword action on animal human health, livelihoods of livestock farmers and food and nutritional security and safety.

\subsection{Misuse of Viral Agents and Biosecurity}

In the present-day advancements in biotechnology, genetic engineering, gene editing, new-generation sequencing and artificial intelligence (AI), there is a common concern about the risk from likely misuse of deadly pathogens of animal or human origin. Massive destruction is possible with the misuse of some of the highly virulent viruses/bacteria/biotoxins as bioterrorism agents upsetting animal and human health and food security and safety. The biowarfare agents can spread widely through animal to animal which may take heavy economic toll due to morbidity, mortality and loss in production. During wartime, particularly involving army operations in difficult hilly or desert terrains, requiring the use of equines or other animal species for transport purpose, natural outbreak of infectious disease or its deliberate introduction by the enemy may cripple the fighting units.

Biosecurity is essential for avoiding the disease entrance across the borders or their spread within defined zones and keep the natural resources (water, soil, feed, food) safe for use. Contemporary farming stresses for a more complete tactic for managing diseases that integrates biosecurity and lay more emphasis on prevention and protection from animal diseases. Introduction of high-producing exotic stock on livestock farms often has inbuilt risk of introducing new viral and other diseases. Biosecurity is a must to safeguard animal health and reduce the risk of new pathogen entry or spread. Biosecurity measures combine the modules of 'external biosecurity' where force is on averting entrance of transboundary animal diseases (TADs) and 'internal biosecurity' where there is focus on stopping disease spread within the country, covering at the zonal and farm level. 
To halt the intrusion of new pathogens through animal trade, the OIE has framed certain standards and guidelines. These guidelines are followed for animals (livestock, poultry, fish, aquatic animals and wildlife) and animal products, including meat and meat products, milk and milk products, egg and egg products, fish and fish products. The standards developed by the OIE from 1995 onwards have been formally recognized by the agreement on the application of sanitary and phytosanitary measures (SPS agreement) of the WTO.

\subsection{Epidemiological Concerns to Shape Research Agenda}

While dealing with the control and management of viral diseases/infections in animal populations, the greatest obstacle is posed by the emergence of antigenic and/or genetic variants of the existing virus types resulting into new types, subtypes, genotypes, clades and lineages, etc. which may not be covered by the existing vaccines in vogue in providing adequate protection leading to vaccine failures. This necessitates redesigning and updating of the vaccines to include the new antigenic variants. Compared to DNA viruses, RNA viruses are more prone to such changes due to their inherent biology, such as segmented genome and possibility of exchange of genes from related virus types resulting into recombination and reassortment. These changes in influenza viruses are described as antigenic drift (gradual minor changes) and antigenic shift (major antigenic alterations). Thus, the need for continuous surveillance and monitoring of virus types in the population and updating of the vaccines becomes a costly and time-consuming proposition. It would be appropriate here to mention the phenomenon of 'antigenic sin' in case of influenza viruses where the infection of a new type of virus may give rise/boost up a detectable HI antibody response against a previous heterologous type/subtype infection. Such findings may confuse in arriving at correct diagnosis based on antibody detection alone.

\subsection{Origin of New Diseases and New Viruses}

Another phenomenon faced by the virologists is the encounter/spread of new infections, diseases from their natural niches (mostly forests, mountains) to new territories and new hosts due to climate change or other conditions leading to close contact of humans and animals with the pathogen(s) under changed conditions. A few examples in this regard include the spread of fox rabies in Europe to human inhabitations due to forcing out of foxes from forest areas as a result of their higher population density. Similarly, in India where jackals serve as important wildlife reservoir of rabies virus, the disease spreads to urban cycle at the beginning of the monsoon when the jackals are forced to come out of their dens (due to filling with rainwater) in the foothills of the Himalayas forcing them to move to nearby human habitations/ villages, thus coming into contact with stray dogs. Fights between dogs and jackals lead to the transmission of the rabies virus from jackals (sylvatic cycle) to urban cycle. In the case of bluetongue disease of sheep and goats, there is now proven 
evidence that the rising temperatures due to climate change have facilitated the spread of the Culicoides vector of the bluetongue virus further northwards into Europe. This had resulted into outbreaks of the disease into new areas/countries.

There is a possibility of exposure of the host to hitherto unknown new viruses from space along with meteorites or 'permafrost' as a result of the melting of snow on polar surfaces due to rising temperatures under climate change scenarios. This will facilitate the release of viruses and other microbes trapped in ice since ages into water streams. Consumption of raw bush meat in African countries is suspected to be the source of new virus pathogens in human.

New viral diseases may arise due to the broadening of the host range of the virus influenced by the genetic makeup of the virus, mutation rates, transmission mechanism such as direct contact transmission or indirect transmission involving vectors (insects, rodents, monkeys, etc.) and reservoir host(s) and the possibility of the new host to acquire receptors of the virus as a result of the coinfection of mammalian or avian hosts with RNA viruses having segmented genome. The best example is provided by influenza viruses when a prevailing human influenza virus and an avian influenza virus coinfect pig, an intermediate host, which has receptors for surface HA antigens of both human and avian viruses. Thus, reassortment of the genetic material of two viruses in the intermediate host could give rise to a new strain that can infect humans leading to pandemics. There have been instances when coinfection of avian virus and influenza viruses of horses resulted in the development of new virulent strains due to genetic shift as a result of genetic reassortment or recombination. The identification of the conditions involving the host, pathogen and the environment, combinations and sequences of events that are likely to change the pattern of infections under a particular set of circumstances, is crucial in understanding and combating viral diseases.

Several factors contribute to the emergence of new viruses, including zoonotic viruses. Some of these include continued increase in global human and livestock populations, thus providing more chances of direct contact between the hosts and the development of modern fast movement and transport by air making it possible to circumnavigate the globe in less than the incubation period of most infectious agents/viruses.

When confronted with a new viral disease, knowledge at the system level, including evolutionary biology, ecosystems, epidemiology and population dynamics of the hosts, vectors and parasites, would be helpful for working out strategies for the prevention of infection/disease. Information on the history, symptoms, lesions and fate of the affected host populations will be of help in the development of diagnostic and differential diagnostic tests (Asokan et al. 2003).

\subsection{Latent Viruses}

Some viruses, including herpes viruses, undergo two phases inside the host, which include the active stage characterized by the multiplication of virus and expression of disease symptoms. In the second phase, i.e. the 'latent phase', the nucleic acid of 
the virus gets integrated with the host cell genome. It is believed that this phase, characterized by the absence of virus multiplication, does no harm to the host. However, whether integration of the virus genetic material/genes with the host genome influences the expression of host genes is a subject for further research. After primary infection, human beta herpesvirus (HHV-6) may establish lifelong latency by integrating with the cellular DNA. The latent virus can be activated under certain conditions, such as immunodeficiency/immunosuppression. Recent studies suggest that HHV-6 is suspected to play a role in the pathogenesis of several diseases of the central nervous system. MicroRNAs produced during active infection and reactivation can prove as biomarkers for this virus. The latent viruses pose challenges in the diagnosis in the dormant phase, control of the disease or establishing disease-free herds.

Immunosuppression due to cortisone therapy, stress of work, pregnancy, higher production and toxicity due to heavy metals, aflatoxin and other mycotoxins is known to make animals more susceptible to diseases. These conditions also reduce the immune response to vaccination. As parasitic infection/infestation leads to debility of the host and intracellular protozoon parasites cause immunosuppression of the host, it is advisable to deworm animals about 2-3 weeks before administering the vaccine. After giving PPR vaccine to sheep and goats, the animals should not be transported or moved to long distances for 3-4 weeks to avoid stress.

\subsection{Naturally Occurring Mild Virus Strains as Vaccines}

There are several examples of naturally occurring mild strains of viruses in the host species or other species which have been used as vaccine candidates. Herpes virus of turkeys (HVT) has been successfully used as vaccine against Marek's disease (a cancerous disease) by administering the vaccine in day-old chicks before they get a chance of infection with the virulent Marek's disease virus (MDV) from the environment. A naturally occurring mild strain of Ranikhet disease virus isolated from pigs in India had been successfully used as vaccine for providing protection against this disease. Similarly, avirulent infectious laryngotracheitis (ILT) virus isolated from apparently healthy chickens in India probably provides natural immunity to birds against the virulent strain of the virus.

\subsection{Interrupting the Contact of Pathogen with the Host}

While confronted with the control of viral disease in poultry, all-out (depopulation)-all-in (repopulation) policy is preferred. It includes destruction of all affected and in contact birds followed by disinfection of the premises and repopulation after keeping the sheds empty for an adequate time to ensure complete freedom from infection under consideration. Depopulation of the susceptible animal species at the international borders up to sufficient distance can be adopted when confronted with a new fatal infectious disease in a neighbouring country, in case no vaccine is 
available to protect the animals. Separation of healthy and infected animals due to natural boundaries, rivers and mountain passes, quarantine or restriction of animal movements can be used with advantage to restrict the spread of the disease. During the equine influenza outbreak in India due to A/Equi-2 virus in 1987 (Uppal and Yadav 1987), setting of isolation camps for sick equines for 2 weeks with provision of potable water and feeding arrangements in the state of Madhya Pradesh in Central India was able to stop the further spread of the disease to the southern states.

In mass vaccination programmes undertaken at national level following OIE pathway, zoning of large countries on the basis of the epidemiological picture of the disease after initial vaccination rounds along with strategic and focused vaccinations at international and interstate borders and animal movement routes to create immune zones and belts of sufficient depth had been a success with saving on vaccine and time taken for getting negative status of the infection. This strategy was followed in India during the rinderpest eradication campaign.

\subsection{Physicochemical Characteristics of the Virus}

Information on the excretion of the virus from body fluids, exhaled air, feathers, etc., is of vital importance in disease management. For example, knowledge that BHV1 virus is excreted intermittently in the semen of bulls can be helpful in using the semen of elite bulls after testing of the semen of the ejaculates found negative by PCR test for freedom from virus. Information that Zika and Ebola viruses may be excreted in the semen for about 6 months is of vital importance in planning appropriate strategies for control of the disease. Similarly, information on the survival of the virus in nature at ambient temperatures, urine, blood, soil, air, water (tap water, canal water), sewerage, vector (if applicable) and common disinfectants can be used with advantage in disease control strategies.

\subsection{Vaccination in the Face of Outbreak}

The objective of vaccination usually had been prophylactic, i.e. to impart specific acquired immunity in the host against a potent pathogen in advance, in case the host animal or human is exposed to that particular pathogen in the near future. Under field conditions, a question is usually asked: if the host is already showing the symptoms of the disease, in that case, should vaccination be undertaken or not? The simple answer is 'no' as the antigen(s) present in the vaccine may make the host more susceptible to the disease as a result of the neutralization of existing humoral antibodies/immunity and diversion of the host immune system to respond to nonimmunogenic proteins in the vaccine will weaken the immune response against the vaccine. However, if the disease occurrence (outbreak) is detected in a closed (animal farm) or defined herd and we are able to detect and diagnose in the beginning, vaccination can be attempted with certain conditions. The first author has experienced handling an outbreak in a dairy farm of the institute, having about a hundred 
cows. On day 1, one cow was reported to be sick with high fever. Examination of the herd revealed that only one cow was having fever as well as lesions of FMD. Only one cow had fever but no lesions in the foot or mouth. Examination of the material collected from the foot-and-mouth lesions of the diseased cow confirmed an FMD virus antigen. The remaining cows in the herd were apparently normal and had no fever or FMD lesions. Two sick cows were separated at the farm. The remaining cows were administered inactivated aluminium hydroxide gel FMD vaccine. The sheds, dung, urine, fodder and feed waste were thoroughly decontaminated with $4 \%$ sodium carbonate $(\mathrm{Na} 2 \mathrm{CO} 3)$ disinfectant before disposal for a week. The interior of the animal sheds including mangers, floor, walls and roof were also disinfected similarly. The milk was properly boiled before feeding to the suckling calves or discarded. This exercise proved effective as no further case of the disease was detected at the infected farm or in other dairy units or experimental animals maintained at the campus. The success may be attributed to the availability of expertise, diagnostic facilities and vaccine, taking prompt decision and action in dealing with the outbreak. Another situation is with rabies subsequent to the bite of a rabid animal; in that case, vaccination is recommended irrespective of the fact whether the animal had been earlier vaccinated against rabies or not. This type of vaccination practiced in rabies is termed as 'therapeutic' vaccination.

\subsection{Vaccination of Pregnant Animals}

In classical textbooks, vaccination in the last quarter of pregnancy is not recommended. One of the reasons is the possibility of abortion while using live vaccines. The possibility of the foetus/newborn developing immune tolerance against the vaccine virus was another consideration. Another view held is that vaccination of pregnant animals in late pregnancy diverts the protein synthesis towards immunoglobulins against vaccine antigens rather than synthesizing the proteins required for the development of the foetus. Thus, it is detrimental for the health of the foetus as well as the newborn. However, there is a need to study the immunobiology of vaccination of animals in the last quarter of pregnancy and its possible effect on the foetus/newborn in response to killed as well as live vaccines.

\subsection{Killed Versus Live Attenuated Vaccines}

Both inactivated and live modified vaccines have merits and demerits. While live vaccines induce long-lasting immunity, are easy to produce at a large scale and require freeze-drying and cold chain during storage and transport, there is remote possibility of the attenuated virus to revert back to become virulent; the killed vaccines on the other hand have weak immunogenicity and require frequent booster doses, costly adjuvants and more space for storage and transport. When the incidence of the disease is high and infection is endemic, live vaccines should be 
preferred. However, killed vaccines may be preferred when the incidence has come down to become negligible after stamping out policy or using live vaccine initially. In case safe live vaccine is not available against a particular virus, the use of killed vaccine is the only alternative left.

When a particular virus affects multiple species, it should be ensured that the attenuated vaccine candidate strain is non-pathogenic to all the susceptible species. In case of FMD virus which affects a number of ruminants and other species, it has been observed that after passage of the virus in cell culture while it became attenuated for one species, it remained pathogenic or became more virulent to other susceptible species. As a result, no safe live vaccine could be developed against FMD.

\subsection{Vaccinated Animals as Carriers of Viral Pathogens}

A common concept is that vaccinated animals pose no risk of spreading the disease to susceptible animals. However, it may not be always so, particularly while using inactivated vaccines and the route of infection is respiratory tract, because inactivated vaccines elicit a poor secretary IgA response which is not adequate to neutralize the virus in the respiratory tract. As a result, the virulent virus if contracted may be harboured in the respiratory tract of the host. Equine influenza and FMD viruses can be cited as examples. The FMD and influenza viruses may persist in the respiratory tract of vaccinated animals due to partial immunity. The FMD virus has been detected in the oesopharyngeal fluid for several months. Similarly, the presence of rabies virus in a few dogs as long as up to 2 years without showing any symptom necessitated the need to revise the policy of recommending vaccination even after the bite of a person by an apparently normal dog.

\subsection{Spillover of Viruses from Laboratory}

To avoid spillover of viruses from research laboratories, strict biosafety and biocontainment procedures need to be followed and adhered to. Viruses can be carried on the hairs of laboratory workers associated with virus research. Hence, as a biosafety precaution, a researcher should not visit an animal farm/unit after working in the laboratory. Similarly, after visiting the experimental or farm animals, one should not visit the laboratory without taking a complete bath with soap and plenty of water. If strict hygienic and biosafety measures are not taken, there are strong chances of transmission of viral infections through farm workers or visitors to animals in rural or urban areas and vice versa.

Application of peptide antibodies and RNAi to inhibit virus multiplication in the host, use of non-coding small RNAs, use of nanotechnology for efficient delivery of drugs and vaccines and developing point-of-care diagnostics and CRISPR-cas gene editing technology are some of the future innovative technologies to be used with advantage in the management and control of viral diseases. 


\subsection{Strategies, Best Practices and Way Forward}

Efficient management of animal viral diseases is a must for accomplishing livestock health protection and promotion to enhance production, productivity and profitability by adopting the suggested action points, strategies, best practices and policy imperatives as follows:

\subsubsection{For Diseases with Eradicated Status}

So far only one viral disease of animals, namely, rinderpest, has been eradicated from India as well as globally. Besides this, African horse sickness (AHS) has also been eradicated from India. Thus, India needs to be prepared for tackling both of these diseases in an emergency situation, preferably using non-infectious diagnostics and vaccines, if these diseases re-emerge. There must be availability of modernized laboratories for quick detection of the etiological agent with skilled manpower. There is need to keep biological material of value, such as vaccine strains, virulent viruses for challenge and antisera under strict biocontainments in BSL III and BSL IV facilities with periodic checkup by FAO/OIE experts/national consultants, or destroyed if so decided. There is also a need to have technical competence and preparedness to develop appropriate vaccine using reverse genetic engineering.

\subsubsection{Capacity Building/Policy Options}

- Veterinary vaccine institutes/biological units in the country should be strengthened and equipped to produce the required diagnostics and combined/polyvalent, thermo-resistant and easy-to-administer vaccines against prevalent major viral and other infectious diseases of livestock.

- Creation of disease-free area/zones with emphasis on export-oriented production.

- Cooperation of village panchayat institutions should be sought for $80-100 \%$ vaccination through incentives to the vaccinators and farmers for getting their animals vaccinated.

\subsubsection{For Other Eradicable Diseases}

- After global eradication of rinderpest, we may succeed in eradicating PPR, a serious disease of small ruminants, caused by a morbillivirus similar to rinderpest virus by 2030 following the OIE pathway and timeline. Similarly, with concerted efforts, adequate vaccine production and funding support, it should be possible to eradicate FMD from India by 2040 adopting the OIE Progressive Control Pathway (PCOP) with significant economic and trade dividends. Presently, India is in the Stage 3 of FAO's COP for the control of FMD (Anonymous 2017-18). 
- Strengthening of the disease diagnostic laboratories at the national, regional and state level with state-of-the-art facilities having desired biosafety and containment facilities of BSL-2/BSL-3 status depending on the type of bioagent being handled is warranted on priority. Other viral diseases, namely, CSF, sheep pox, goat pox, fowl pox and rabies, may also be included in national eradication programmes as potent vaccines are available against these.

- As India has long porous land borders with the neighbouring countries all around, there is always threat of transboundary diseases. Ideally disease control/eradication programmes should be taken in network mode involving all the neighbouring SAARC/ASEAN countries. A venture fund should be created by the participating countries for routine programmes to meet emergency situations in the face of disease outbreaks.

- Farmers need to be updated on useful livestock technologies, best practices and innovations in animal health, nutrition and management. This will require strengthening of extension services for livestock, poultry and fishery in PPP mode using modern ICT tools.

- A comprehensive livestock health policy should be developed at the national and state level.

- Capacity building for developing cold chain; testing the quality of vaccines, medicines, mineral mixture and residues in livestock feed; and production of disease-free semen, chicks, and fingerlings.

- The success story of rinderpest eradication and FMD-CP needs to be replicated for PPR, classical swine fever, HS, brucellosis and other dreadful diseases of livestock and poultry.

- A state-level zoonoses coordination committee with subcommittees at the district level should be constituted under the umbrella of the Department of Animal Husbandry GoI to bring about close association among veterinarians, medical professionals, wildlife experts and other related departments focusing on the 'One World, One Health' concept for efficient handling of the newly emerging and re-emerging deadly viral and other infectious diseases having zoonotic significance.

\subsection{Epilogue}

Animal disease surveillance is critical for the welfare and sustainability of healthy livestock productivity systems of any country, as the threat of infectious diseases is large as well as diverse and dynamic. Preparedness and combating the prevailing emerging, re-emerging and transboundary diseases require sound monitoring and precision detection systems that are flexible and adaptable under field conditions. There is also an international obligation for OIE reportable diseases of high importance from the point of view of trading in animals and animal products harbouring potent infectious agents/pathogens by all member countries of WTO. It is the right time to apply developed diagnostics and molecular detection tools at the field stage to ensure fast detection and confirmation of pathogens capable of causing diseases 
in humans and animals. This must be accompanied by national-level disease surveillance, monitoring and networking to enable an early warning system for infectious diseases based on forecasting (Saminathan et al. 2016). Due priority is also required for application of new vaccines, developing vaccine delivery systems and adopting prudent vaccination programmes and immunomodulatory and effective therapeutic modalities, which would help in devising timely prevention and control strategies against viral and other infectious diseases. Besides these, good management and standard biosecurity measures/practices and appropriate hygienic and zoo-sanitary and quarantine measures should be observed. Moreover, on-the-spot control and checking the spread of pathogens and adequate trade restrictions as envisaged under the SPS agreement of WTO also need to be followed. A holistic vision is required for timely implementation of these concepts and strategies along with strengthening of various multidimensional research and development programmes backed with appropriate funding resources. These measures will greatly help minimize disease incidences and outbreaks and lessen economic burdens due to infectious animal diseases and boost livestock and poultry health, reproduction and production to strengthen sustainable growth of livestock and poultry industry. Reduction in pandemic threats and public health concerns eventually lead to improvement in the socioeconomic status and welfare of the society at large.

Acknowledgements All the authors of the manuscript thank and acknowledge their respective universities and institutes.

Conflict of Interest There is no conflict of interest.

\section{References}

Anonymous (2011) Annual report, project directorate on animal disease monitoring and surveillance, Bangalore, India, pp 1-87

Anonymous (2013) Annual report, project directorate on animal disease monitoring and surveillance, Bangalore, India, pp 1-98

Anonymous (2017-18) Annual report, ICAR-directorate of foot-and-mouth disease, Mukteswar, India, pp 1-88

Asokan GV, George A, Vasanthan A, Prabhakaran V, Prabhakaran SK (2003) Epidemiology of emerging viral zoonoses. Indian J Anim Sci 73(4):337-341

Chakraborty S, Kumar A, Tiwari R, Rahal A, Malik YS, Dhama K, Amarpal, Prasad M (2014) Advances in diagnosis of respiratory diseases of small ruminants: a review. Vet Med Int 2014:508304, 16. https://doi.org/10.1155/2014/508304

Dhama K, Chakraborty S, Tiwari R, Verma AK, Saminathan M, Amarpal MYS, Khan RU, Nikousefat Z, Javdani M (2014) A concept paper on novel technologies boosting production and safeguarding health of humans and animals. Res Opin Anim Vet Sci 4(7):353-370

Dhama K, Karthik K, Khandia R, Chakraborty S, Munjal A, Latheef SK, Kumar D, Ramakrishnan MA, Malik YS, Singh R, Malik SVS, Singh RK, Chaicumpa W (2018) Advances in designing and developing vaccines, drugs, and therapies to counter Ebola virus. Front Immunol 9:1803. https://doi.org/10.3389/fimmu.2018.01803 
Hallen JHB, Mcleod K, Charles JG, Keer HC, Allijan MM (1871) The cattle plague commission report to government of India. Calcutta Publication, Calcutta, pp 1-999

Joshi VG, Dighe VD, Thakuria D, Malik YS, Kumar S (2013) Multiple antigenic peptide (MAP): a synthetic peptide dendrimer for diagnostic, antiviral and vaccine strategies for emerging and re-emerging viral diseases. Indian J Virol 24(3):312-320

Kumar S (1976) African horse sickness. ICAR Tech Bull 15:1-34

Loeffler F, Frosch P (1898) Zentralbl, Bacteriol, Parasitenkd, Infektionskr. Hyg Abt 1 Orig 28:371

Malik YS, Dhama K, Singh RK (2018) Emerging and zoonotic virus challenges of developing nations. Open Virol J 12:42-43. https://doi.org/10.2174/1874357901812010042

Reed W, Carroll J, Agramonte A, Lazear J (1901) Senate Documents 66(822):156

Rout M, Kumar S, Malik YS (2014) DIVA vaccines and companion diagnostics with relevance in animal disease eradication. J Immunol Immunopathol 16(1\&2):12-19

Rout M, Bhat S, Malik YS, Chauhan RS (2018) Biosensor's use in detection of animal diseases and enhancing production. J Immunoass Immunochem 20(2):74-82

Saminathan M, Rana R, Ramakrishnan MA, Karthik K, Malik YS, Dhama K (2016) Prevalence, diagnosis, management and control of important diseases of ruminants with special reference to Indian scenario. J Exp Biol Agric Sci 4(3S):3338-3367. https://doi.org/10.18006/2016.4 (3s).338.367

Singh RK, Dhama K, Karthik K, Tiwari R, Khandia R, Munjal A, Iqbal HM, Malik YS, BuenoMarí R (2017) Advances in diagnosis, surveillance, and monitoring of Zika virus: an update. Front Microbiol. https://doi.org/10.3389/fmicb.2017.02677

Singh RK, Dhama K, Karthik K, Khandia R, Munjal A, Khurana SK, Chakraborty S, Malik YS, Virmani N, Singh R, Tripathi BN, Munir M, vander Kolk JH (2018) A comprehensive review on equine influenza virus: etiology, epidemiology, pathobiology, advances in developing diagnostics, vaccines and control strategies. Front Microbiol. https://doi.org/10.3389/ fmicb.2018.01941

Tripathi BN, Kumar N, Barua S (2018) Peste des Petits ruminants: sheep and goat plague. Today and Tomorrow's Printers and Publishers, New Delhi, pp 1-180

Uppal PK (2011) FAO sponsored final project report "National testimonies" under the Global Rinderpest Eradication Programme (GREP)-(GCP/GLO/302/EC) pp 1-134

Uppal PK, Yadav MP (1987) Outbreak of equine influenza in India. Vet Rec 121:567-570

Uppal PK, Yadav MP (1989) Occurrence of equine infectious Anaemia in India. Vet Rec 124:514-515

Yadav MP (2011) FAO sponsored final project report on "Laboratory contributions for rinderpest eradication in India" under the Global Rinderpest Eradication Programme (GREP)-(GCP/ GLO/302/EC), pp 1-58

Yadav MP, Uppal PK, Mumford JA (1993) Physico-chemical and biological characterization of a/ Equi-2 virus isolated from 1987 equine influenza epidemic in India. Int J Anim Sci 8:93-98

Yadav MP, Uppal PK, Rao JR (2016) Animal sciences. In: Singh RB (ed) 100 years of agricultural sciences in India. NAAS, New Delhi, pp 158-258 